\title{
Multiwavelength observations of isolated neutron stars as a tool to probe the properties of their surfaces
}

\author{
G. G. Pavlov \\ The Pennsylvania State University, 525 Davey Lab, University Park, PA 16802, USA; \\ pavlov@astro.psu.edu \\ and \\ V. E. Zavlin, J. Trümper, and R. Neuhäuser \\ Max-Planck-Institut für Extraterrestrische Physik, D-85740 Garching, Germany; \\ zavlin@rosat.mpe-garching.mpg.de
}

\begin{abstract}
We show that an analysis of multiwavelength observations of isolated neutron stars based on neutron star atmosphere models can be used not only to evaluate the neutron star effective temperature, but also to determine chemical composition of its surface. To demonstrate how this new method can be applied to a specific object, we chose the old isolated neutron star candidate RXJ1856.5-3754, whose soft X-ray radiation has been studied recently by Walter, Wolk, \& Neuhäuser (1996). We fitted the soft X-ray spectrum of this object with neutron star atmosphere models of different chemical compositions and used these fits to calculate the source spectrum over a broad wavelength range. We showed, in particular, that the optical/UV flux expected from this object depends drastically on the composition of its surface. In particular, the neutron star covered with hydrogen would be 5-6 magnitudes brighter than the neutron star with an iron surface. The object should also be observable with EUVE; the EUV flux is expected to be almost twice higher for the iron surface than for the hydrogen one. Thus, multiwavelength observations of this object would enable one to examine, for the first time, chemical composition of the neutron star surface. The method proposed can be applied to other nearby isolated neutron stars.
\end{abstract}

Subject headings: stars: atmospheres — stars: individual (RXJ1856.5-3754) — stars: neutron - ultraviolet: stars — X-rays: stars

\section{Introduction}

It follows from both extrapolating pulsar birthrates (Narayan \& Ostriker 1990) and from the number of supernovae required to account for the heavy element abundance (Arnett et al. 1989) 
that the Galaxy is populated by about $10^{8}-10^{9}$ neutron stars (NSs). However, only $\sim 10^{-6}-10^{-5}$ of this amount has been observed so far, mainly as radio pulsars. Thus, one can expect that there exists a vast population of nonpulsating NSs which could be observed as faint sources of thermal radiation. It has recently been claimed by Walter, Wolk, \& Neuhäuser (1996; hereafter WWN96) that the bright, nonpulsating, soft ROSAT X-ray source RXJ1856.5-3754 is a viable candidate to be a member of this population.

WWN96 found that (a) a blackbody fit of the spectrum detected with the ROSAT Positional Sensitive Proportional Counter (PSPC) (count rate $3.62 \pm 0.03 \mathrm{~s}^{-1}$ ) yields an apparent blackbody temperature of $6.6 \times 10^{5} \mathrm{~K}$, a hydrogen column density of $1.4 \times 10^{20} \mathrm{~cm}^{-2}$, and an emitting area of $480(d / 100 \mathrm{pc}) \mathrm{km}^{2}$; (b) other standard models do not give better fits (see also Neuhäuser et al. 1996 for details); (c) the ROSAT High Resolution Imager (HRI) count rate is $0.56 \pm 0.005$ $\mathrm{s}^{-1}$; (d) there is no evidence for X-ray variability in this source; and (e) the object is fainter than $V \simeq 23$ in the visual band. No radio, UV, or gamma radiation have been detected. The source is projected onto the $\mathrm{R} \mathrm{CrA}$ dark molecular cloud, at a distance of $\sim 120$ pc (Wang 1994). The observed X-ray extinction allows one to assume that the source is closer than the cloud.

The inferred temperature looks surprisingly high for this presumably very old NS (see arguments in WWN96), although it may be caused by enhanced accretion of interstellar matter if the NS relative motion is slow enough. However, the real spectrum of the NS thermal emission may differ substantially from the Planck spectrum due to the presence of an atmosphere on the NS surface (e. g., Pavlov et al. 1995), and the actual shape of the spectrum strongly depends on chemical composition of the emitting layers (Rajagopal \& Romani 1996; Zavlin, Pavlov, \& Shibanov 1996, hereafter ZPS96). Fitting observed spectra with the model atmosphere spectra may result in quite different effective temperature $T_{\text {eff }}$ and ratio $R / d$ of the size of emitting region to the distance.

The chemical composition of the NS surface depends on various effects. Due to strong gravitation at the NS surface, the elements there are separated in accordance with their atomic weights so that the lightest element resides at the very surface and forms the atmosphere, whereas heavier elements sink down. Thus, if an amount of hydrogen $(\mathrm{H})$ is present in the outer layers (e. g., due to accretion of the interstellar matter), one can expect a pure $\mathrm{H}$ atmosphere. If during the NS life time there were no substantial accretion of the H-rich matter, or the hydrogen were burnt or ejected from the surface, the atmosphere may be comprised of a heavier element. In principle, it might be helium (He) or carbon, although the most natural choice seems to be iron (Fe) formed in the core of the supernova progenitor star. The $\mathrm{H}$ and $\mathrm{He}$ atmospheres emit spectra which are much harder in the Wien tail than the blackbody (BB) spectrum because hotter deep layers are seen at high frequencies where the spectral opacity is lower. In this case, the best-fit atmosphere effective temperature is considerably lower than the BB temperature, whereas the $R / d$ ratio is larger than for the $\mathrm{BB}$ fit. On the contrary, in the case of the Fe atmosphere the overall shape of the spectrum is not so drastically different from that of the BB model, although local deviations may be quite substantial due to numerous spectral lines and photoionization edges. 
The chemical composition of the NS surface could be determined by the analysis of its soft $\mathrm{X}$-ray spectrum. However, spectral resolution and sensitivity of current soft X-ray detectors is not high enough to firmly detect spectral features in the most interesting range $\sim 0.1-1 \mathrm{keV}$. On the other hand, this range is so short that the shape of the continuum can be fitted with quite different models. Thus, it is desirable to measure the NS flux over a broader range. Since the NS thermal flux is intrinsically very low at medium and hard X-rays, and it is severely suppressed by the interstellar extinction at EUV, the most perspective would be optical/UV observations $(E \simeq 2-10 \mathrm{eV})$. Although NSs are extremely faint in this range (typical expected magnitudes are $\sim 26-28$ at $d \sim 100 \mathrm{pc}$ ), broad-band photometry of these sources is quite feasible with the Hubble Space Telescope (HST) and the largest ground-based telescopes (Pavlov 1992).

So far, optical/UV thermal radiation has been apparently detected from only two active pulsars, PSR B1929+10 and (more questionable) PSR B0950+08 (Pavlov et al. 1996); optical radiation from younger, 'middle-aged' pulsars Geminga and PSR B0656+14 is most likely of a non-thermal origin (Bignami et al. 1996; Pavlov et al. 1996). The pulsars B1929+10 and B0950+08 have relatively strong magnetic fields $\left(\sim 10^{12} \mathrm{G}\right)$, which precludes analysis of the atmosphere chemical composition because no reliable opacities have been calculated for elements other than hydrogen for strongly magnetized dense plasmas. On the contrary, for very old NSs, where the magnetic field is believed to have been decayed and rotation has slowed down so that the NSs cannot manifest themselves as active pulsars, the chemical composition can be analyzed with the use of non-magnetic atmosphere models.

In the present paper we fit the ROSAT PSPC spectra of the NS candidate RXJ1856.5-3754, which seems to be sufficiently old and does not show pulsar activity, with the $\mathrm{H}, \mathrm{He}$, and Fe atmosphere models developed by ZPS96. We use these fits to calculate the IR through X-ray fluxes expected from this source, assuming that it is indeed an old, isolated NS with low magnetic field. We show that the detected optical flux (and the distance to the object) would immediately allow us to distinguish between light-element ( $\mathrm{H}$ or $\mathrm{He}$ ) and heavy-element composition. We show that for any assumptions on the chemical composition the object should be observable with the $E U V E$ Deep Survey (DS) instrument in a relatively short exposure time. The most dramatic effect of the chemical composition is expected at optical wavelengths, where the NS covered with hydrogen would be about 5-6 magnitudes brighter than the NS with an iron surface.

\section{PSPC fits and the sourse flux in a broad energy range}

We re-analized the PSPC data using the EXSAS software (Zimmermann et al. 1994). The source in the PSPC image is 14.6 off-axis, and its measured (off-axis) count rate is $3.23 \pm 0.04$

$\mathrm{s}^{-1}$, which corresponds to the corrected (on-axis) count rate of $3.64 \pm 0.04 \mathrm{~s}^{-1}$, in agreement with WWN96.

We binned the observed count rate spectrum into 19 energy channels between approximately 
Table 1: PSPC fits, corresponding $V$ magnitudes, and count rates for other detectors

\begin{tabular}{ccccccccc}
\hline \hline Model & $\begin{array}{c}n_{H} \\
\left(10^{20} \mathrm{~cm}^{-2}\right)\end{array}$ & $\begin{array}{c}T_{\text {eff }} \\
\left(10^{5} \mathrm{~K}\right)\end{array}$ & $\begin{array}{c}d \\
(\mathrm{pc})\end{array}$ & $\begin{array}{c}\chi_{\nu}^{2} \\
\mathrm{~V}\end{array}$ & $\begin{array}{c}\mathrm{HRI}^{\mathrm{a}} \\
\left(\mathrm{s}^{-1}\right)\end{array}$ & $\begin{array}{c}\mathrm{DS}^{\mathrm{b}} \\
\left(\mathrm{s}^{-1}\right)\end{array}$ & $\begin{array}{c}\text { Scanner }^{\mathrm{c}} \\
\left(\mathrm{s}^{-1}\right)\end{array}$ \\
\hline $\mathrm{H}$ & $2.14 \pm 0.08$ & $2.04 \pm 0.36$ & $4.8 \pm 1.1$ & 0.94 & 21.8 & 0.60 & 0.046 & 0.045 \\
$\mathrm{He}$ & $2.30 \pm 0.08$ & $1.95 \pm 0.31$ & $3.8 \pm 1.0$ & 1.24 & 20.8 & 0.60 & 0.043 & 0.043 \\
$\mathrm{Fe}$ & $0.95 \pm 0.04$ & $9.78 \pm 1.45$ & $216 \pm 33$ & 0.68 & 27.5 & 0.69 & 0.087 & 0.065 \\
blackbody $^{\mathrm{d}}$ & $1.49 \pm 0.03$ & $8.51 \pm 1.43$ & $158 \pm 26$ & 0.80 & 26.8 & 0.61 & 0.053 & 0.047 \\
H magnetic $^{\mathrm{d}}$ & $2.21 \pm 0.07$ & $2.88 \pm 0.43$ & $12.1 \pm 1.3$ & 1.32 & 23.3 & 0.60 & 0.044 & 0.043 \\
\hline
\end{tabular}

${ }^{a}$ Predicted ROSAT HRI count rate

${ }^{b}$ Count rate predicted for the $E U V E$ Deep Survey instrument with the Lexan/B filter

${ }^{c}$ Count rate predicted for the EUVE Scanner A/B with the Lexan/B filter

${ }^{d}$ For $B=1 \times 10^{12} \mathrm{G}$

0.1 and $1.0 \mathrm{keV}$ (no source counts were detected above $1 \mathrm{keV}$ ) and fitted it with the H, He, and Fe atmosphere models of ZPS96, and with the BB spectrum. The best-fit parameters (effective hydrogen column density $n_{H}$, unredshifted surface effective temperature $T_{\text {eff }}$, distance to the source $d$ ), and reduced $\chi_{\nu}^{2}$ (for 16 degrees of freedom) are presented in Table 1 . We assumed standard NS mass $M=1.4 M_{\odot}$ and radius $R=10 \mathrm{~km}$ (which corresponds to the apparent radius $R_{\infty}=g_{r}^{-1} R=13 \mathrm{~km}$, where $\left.g_{r}=\left[1-\left(2 G M / R c^{2}\right)\right]^{1 / 2}\right)$. Although the BB temperature and $n_{H}$ are essentially the same as obtained by WWN96 (notice that the unredshifted temperature $T_{\text {eff }}$ is higher than the apparent temperature $T_{\text {eff, } \infty}=g_{r} T_{\text {eff }}$ presented by WWN96), the formal quality of our fit is much better (minimum $\chi_{\nu}^{2}=0.80$ vs. 2.31 ), most likely because we chose another binning of the PSPC count spectrum.

Since the spectra emitted by the $\mathrm{H}$ and He atmospheres are substantially harder, in the ROS AT range, than the BB spectrum with the same effective temperature, we obtained quite different fitting parameters for the atmosphere model fits. For example, $T_{\text {eff }}^{\mathrm{BB}} / T_{\text {eff }}^{\mathrm{H}}=4.17$ and $d^{\mathrm{BB}} / d^{\mathrm{H}}=32.8$. On the other hand, the difference between the parameters obtained for the $\mathrm{BB}$ and Fe atmosphere fits is not so dramatic because the Fe atmosphere spectrum is close, on average, to the BB spectrum of the same temperature (ZPS96). Moreover, the Fe model spectrum is even slightly softer than $\mathrm{BB}$ in the ROSAT range due to strong bound-free absorption, which leads to higher $T_{\text {eff }}$ and $d$. Despite the significant difference in the best-fit parameters (particularly, $T_{\text {eff }}$ and $d$ ), the fits look very similar. From the viewpoint of formal statistics, all the four fits are acceptable, i. e., one cannot choose the most acceptable model without invoking additional arguments.

The projections of the three-dimensional confidence regions onto the $T_{\text {eff }}-n_{H}$ plane, together with the lines of constant most probable distances, are shown in Figure 1 for the four spectral 
models. We see that the confidence regions for $\mathrm{H}$ and He models overlap with each other, being very distant from those for the $\mathrm{Fe}$ and $\mathrm{BB}$ models.
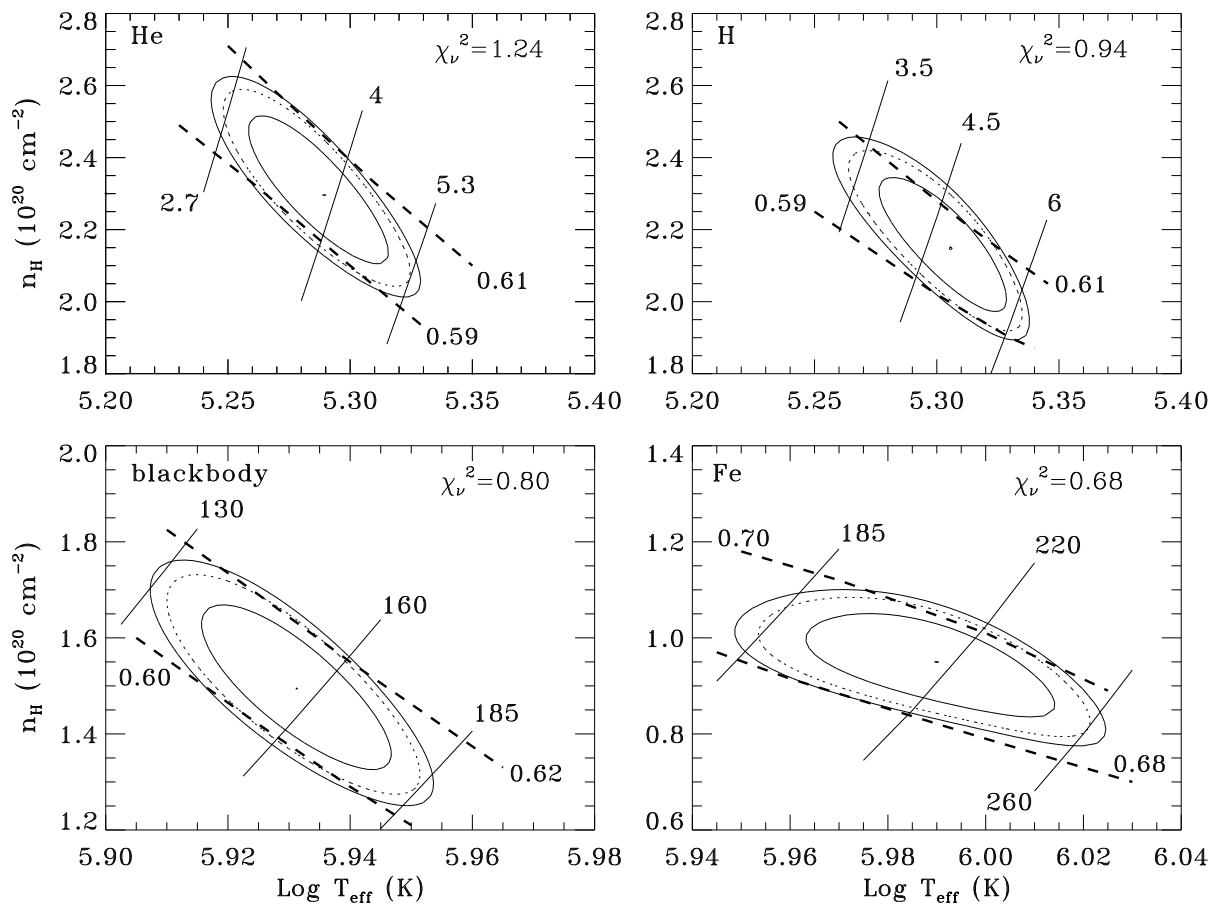

Fig. 1. Confidence contours for the helium, hydrogen, and iron atmosphere models, and the blackbody model, at $68 \%(1 \sigma), 90 \%$ and $99 \%$ confidence levels. Nearly straight solid lines are the lines of constant most probable distance $d$ (in pc). The thick dashed lines correspond to the constant HRI count rates (figures near the curves, in $\mathrm{s}^{-1}$ ).

Since the energy dependence of the HRI and PSPC effective areas are different, the HRI observations of the same object can provide an additional opportunity to examine the models for its radiation. We re-analyzed the HRI image and obtained the source count rate $0.56 \pm 0.01$ $\mathrm{s}^{-1}$. The HRI count rates corresponding to the best PSPC fit models are presented in Table 1, and the curves of constant HRI count rates are shown in Figure 1. We see that the model HRI count rates exceed the measured one by $5-9 \%$ for the $\mathrm{H}$ and He models, $7-11 \%$ for the BB model, and $20-25 \%$ for the Fe model. The systematic uncertainty of the HRI effective area at low energies, $0.1-0.5 \mathrm{keV}$, which give the main contribution to the HRI count rate, may reach $5-7 \%$ (B. Aschenbach, private communication). Since the PSPC response is also not free from systematic errors at low energies, one cannot exclude that the $\mathrm{H}$ and $\mathrm{He}$ atmosphere models are consistent with the HRI data, whereas the Fe model seems to give a too high HRI count rate.

To demonstrate how observations of this object in other spectral bands can be used to discriminate between the source models, we calculated the spectral fluxes, both attenuated and non-attenuated by the interstellar extinction, for the best PSPC fit models over the broad photon energy range, $1-1000 \mathrm{eV}$ (Fig. 2). To calculate the extinction at IR/optical/UV energies, 
we use the approximate relation between the hydrogen column density and the color excess: $E(B-V)=n_{H} /\left(5.8 \times 10^{21} \mathrm{~cm}^{-2}\right)$ - see Savage \& Mathis (1979). In the range of $0.1-1.0$ $\mathrm{keV}$ all the attenuated spectra are very similar to each other, especially for the $\mathrm{H}$, He, and BB models, in accordance with approximately the same quality of the PSPC fits. In the range of $\simeq 10-80 \mathrm{eV}$ the fluxes are unobservable for all the models because of the interstellar absorption. They are observable in the high-energy part of the $E U V E$ spectral range, $\simeq 100-200 \mathrm{eV}$, which considerably overlaps with the ROSAT soft channels. We evaluated expected count rates for the $E U V E$ DS and EUVE Scanners A/B with the Lexan/B filter for different models (see Table 1). The count rates are not much different for the $\mathrm{H}, \mathrm{He}$, and $\mathrm{BB}$ models, $\simeq 0.04-0.05 \mathrm{~s}^{-1}$, whereas for the Fe model the count rate is higher, particularly for the DS instrument. A $3 \sigma$ upper limit of $0.066 \mathrm{~s}^{-1}$, reported by WWN96 for a short (1,001 s) Scanner exposure, is too high to discriminate between the models (although the Fe atmosphere count rate almost coincides with the limit). A longer, $\sim 20-30 \mathrm{ksec}$, observation with the EUVE DS is needed to detect $\sim 1000$ source counts and to draw a convincing conclusion on the source nature.

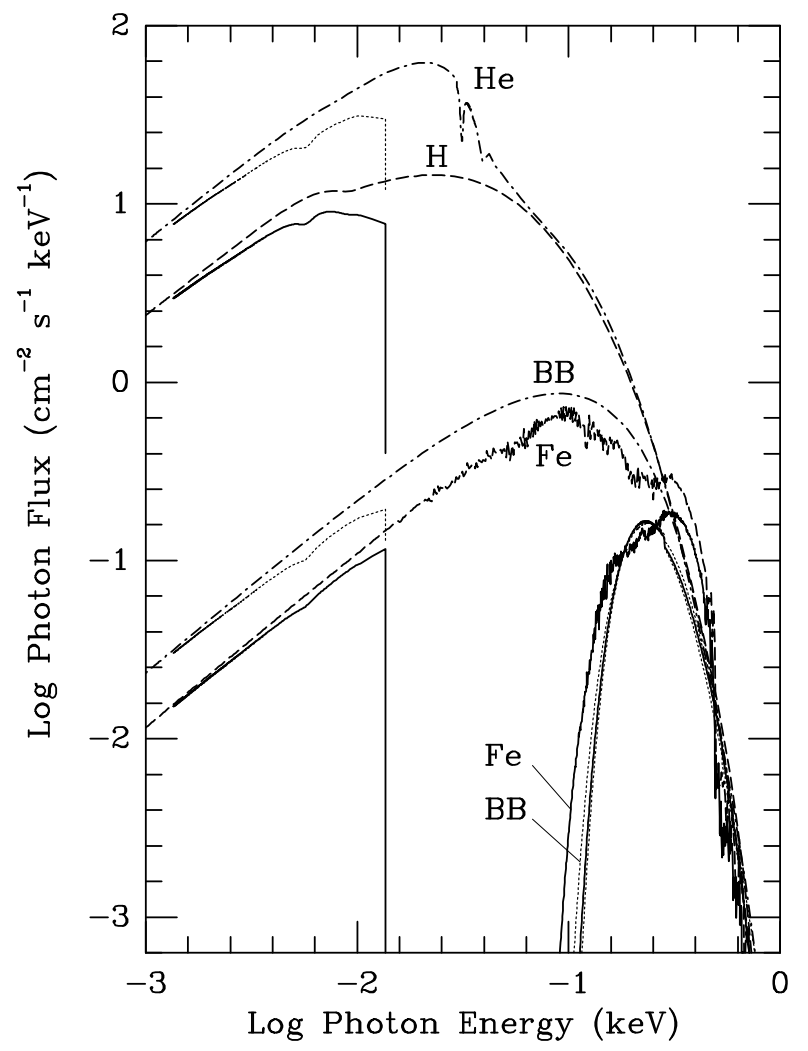

Fig. 2. Photon spectral fluxes for four models in the range of $1-1000 \mathrm{eV}$ with and without allowance for interstellar absorption.

Much more striking difference between the model fluxes is seen in the IR/optical/UV range $(1-10 \mathrm{eV})$ : for instance, the source flux is $6-7$ magnitudes brighter for the $\mathrm{H}$ and He models than for the Fe model (see Table 1). The reason is that a $40-50$ times smaller distance (at a 
given emitting area) is needed for the $\mathrm{H} / \mathrm{He}$ models to provide the same soft-X-ray flux at $4-5$ times lower effective temperature. The spectra at the low energies follow the Rayleigh-Jeans law, $F_{\nu} \propto \nu T(R / d)^{2}$, so that the flux for the $\mathrm{H} / \mathrm{He}$ models is $400-500$ times higher than for the Fe model. Since the effect of extinction is not very significant, the source is expected to be the brightest at UV frequencies.

\section{Discussion and conclusions}

The $V$ magnitude predicted for the $\mathrm{H} / \mathrm{He}$ models is about 20-22, which is brighter than the limit of $V=23$ reported by WWN96. If this limit is correct, then the non-magnetic $\mathrm{H} / \mathrm{He}$ models can be excluded. One more striking feature of the $\mathrm{H} / \mathrm{He}$ atmosphere models is a very small $d / R$ ratio - for instance, for $R=10 \mathrm{~km}$ the distance would be in a range of $3.1-6.3 \mathrm{pc}$, at a $99 \%$ confidence level, if the NS is covered with hydrogen. For the expected local NS density, $\sim 10^{-4}-10^{-3} \mathrm{pc}^{-3}$, the probability to find a NS at such a distance is low (e. g, the probability to find one NS at a distance of $5 \mathrm{pc}$ from the Sun is $\sim 0.05-0.5)$. In principle, the NS radius may be larger than $10 \mathrm{~km}$; for example, $R \simeq 15 \mathrm{~km}$ for a NS of the mass $1.3 M_{\odot}$ for a hard equation of state (e. g., Shapiro \& Teukolsky 1983). In this case, the distance would become larger by a factor of 1.5. The distances following from the $\mathrm{H} / \mathrm{He}$ atmosphere models can be easily measured with

the HST even for such a faint object. On the contrary, if we adopt the Fe atmosphere model, the distance of $180-270 \mathrm{pc}$ would be even larger than for the BB fit, considerably exceeding $120 \mathrm{pc}$ to the R CrA molecular cloud. This also seems unlikely because the total gas column density of the cloud in this region, $\sim 5 \times 10^{20} \mathrm{~cm}^{-2}$, is substantially greater than the $99 \%$ upper limit of $n_{H} \simeq 1.1 \times 10^{20} \mathrm{~cm}^{-2}$.

Thus, at the present stage, the situation looks puzzling - light element models give uncomfortably small distances and optical magnitudes brighter than the reported limit, whereas the Fe model results in a distance larger than acceptable, if the NS is foreground to the R CrA dark cloud. This conclusion, however, is based on the assumption that the magnetic field at the NS surface does not exceed $10^{9}-19^{10} \mathrm{G}$ (otherwise the non-magnetic atmosphere models are inapplicable). Although there have been many speculations about the field decay in isolated NSs (e. g., Itoh et al. 1995, and references therein), this idea is not firmly supported by observations to date. The main argument against the presence of high magnetic fields in RXJ1856.5-3754 is that no significant pulsations have been found in the ROSAT data (Becker 1996). If the lack of pulsations is confirmed, it still might be consistent with the presence of high magnetic fields if the rotation axis coincides with the magnetic axis or with the line-of-sight. The former is not unexpected for an old NS; the probability of the latter is very low. Fitting the observed spectra with the magnetic $\mathrm{H}$ atmosphere models may not be as definitive as with the nonmagnetic ones because we know nothing about the strength and geometry of the field which, in turn, affects the distribution of temperature over the NS surface. Besides, the available magnetic models may not be accurate at relatively low temperatures (Pavlov et al. 1995). An example of the magnetic 
$\mathrm{H}$ atmosphere fit (assuming uniform temperature and $B=1 \times 10^{12} \mathrm{G}$ ) is presented in Table 1 . Generally, magnetic fits result in higher effective temperatures, greater distances, $d \sim 10-30 \mathrm{pc}$, and fainter optical magnitudes, $V \simeq 23-25$, than the nonmagnetic $\mathrm{H} / \mathrm{He}$ atmosphere fits. Although the distances still look very small, such parameters do not contradict to the data available.

The most reliable way to understand the nature of RXJ1856.5-3754 is to conduct deep optical/UV observations of a relatively wide field around its X-ray position. It should be noted that although the X-ray position of a star detected in the HRI pointed observation is coincident, within $0.7 \pm 2$ !' 6 , with its HST GSC position (WWN96), the latter may itself be in error by a few arcseconds (e. g., because of a large proper motion). Hence, the HRI position of the NS may be less accurate than $2^{\prime \prime}$, as it was adopted by WWN96. In addition, if the distance to the NS is indeed as low as a few parsec, one should expect a large proper motion of the object. For instance, if one adopts the NS tangential velocity of $300 \mathrm{~km} / \mathrm{s}$, a typical value for radio pulsars, and a distance of $10 \mathrm{pc}$, then the proper motion would be $\mu=6^{\prime \prime} 2 v_{300} d_{10}^{-1} \mathrm{yr}^{-1}$. The proper motion might be limited by the relatively small mean offset, $\simeq 26^{\prime \prime}$, between the Einstein IPC slew survey position and the HRI position measured 14 years later. This limit, however, is not very stringent because of the large uncertainty, $72^{\prime \prime}$ at $90 \%$ confidence level, of the Einsten position: the resulting proper motion can be estimated as $\mu=2^{\prime \prime} \pm 5^{\prime \prime} \mathrm{yr}^{-1}$. Thus, one cannot exclude that the object could travel $\sim 10^{\prime \prime}$ during 1.4 years between the HRI and optical observations, so that the reported limit of $V=23$ should be considered with caution, and a wider field should be carefully investigated before making final conclusions. To prove that a faint object in the field is indeed the NS, the color of the object must be examined - NSs of expected temperatures should be very blue.

If a more accurate investigation of the field results in detection of this source with a $V$ magnitude of about $21-22$, and a distance of $3-7 \mathrm{pc}$, the conclusion would be quite unambiguous: the NS has an $\mathrm{H}$ or He atmosphere and a very low magnetic field. If the magnitude is slightly fainter, about 24, and the distance larger, $10-30 \mathrm{pc}$, we would argue for the magnetic $\mathrm{H}$ atmosphere model and conclude that magnetic and rotation axes coincide with each other, or the rotation axis is co-aligned with the line of sight. Finally, if the magnitude is as faint as $V \simeq 27-28$ and/or the distance exceeds $\sim 150-200 \mathrm{pc}$, then we conclude that the atmosphere consists of Fe or another heavy element. In this case we should also conclude that the NS is seen through a hole in the cloud R CrA, unless the distance to the cloud was underestimated. Thus, optical observations of this object would enable one, for the first time, to probe chemical composition of surface layers of NSs and to firmly evaluate their temperature. Obviously, the same approach, i. e., combined analysis of the X-ray and optical/UV spectra with the use of the NS atmosphere models, can be applied to other similar objects.

We thank Fred Walter, Werner Becker, Bernd Aschenbach, Stuart Bowyer, and the referee for useful discussions. The ROSAT project is supported by the BMBF and the Max-Planck-Society. G. P is grateful to the Max-Planck-Institut für Extraterrestrische Physik for hospitality; his 
work was partially supported by NASA grant NAG5-2807 and INTAS grant 94-3834. V. Z. acknowledges Max-Planck fellowship and the INTAS grant.

\section{REFERENCES}

Arnett, W. D., Schramm, D. N., \& Truran, J. W. 1989, ApJ, 339, L25

Becker, W. 1996, private communication

Bignami, G. F., et al. 1996, ApJ,456, L111

Itoh, N., Kotouda, T., \& Hiraki, K. 1995, ApJ, 455, 244

Narayan, R., \& Ostriker, J. P. 1990, ApJ, 352, 222

Neuhäuser, R., Walter, F. M., \& Wolk, S. J., 1996, In Proc. of the Workshop on Supersoft X-ray Sources, Lecture Notes in Physics, ed. J. Greiner (Berlin: Springer), 279

Pavlov, G. G. 1992, in Proc. IAU Coll. 122, "The Magnetospheric Structure and Emission Mechanisms of Radio Pulsars", ed. T. H. Hankins, J. M. Rankin, \& J. Gil, p. 220

Pavlov, G. G., Shibanov, Yu. A., Zavlin, V. E., \& Meyer R. D. 1995, in The Lives of the Neutron Stars, ed. M. A. Alpar, Ü. Kiziloğlu, \& J. van Paradijs (Dordrecht: Kluwer), 71

Pavlov G. G., Stringfellow G. S., \& Córdova, F. A., 1996, ApJ, 467, 370

Rajagopal, M., \& Romani, R. W. 1996, ApJ, 461, 327

Savage, B. D., \& Mathis, J. S. 1979, ARA\&A, 17, 73

Shapiro, S., \& Teukolsky, S. 1983, Black Holes, White Dwarfs and Neutron Stars (Wiley: New York)

Walter, F. M., Wolk, S. J., \& Neuhäuser, R. (WWN96), 1996, Nature, 379, 233

Wang, Q. D. 1994, in The Soft X-Ray Cosmos, ed: E. M. Schlegel, R. Petre, AIP Conf. Proc., Vol. 313 (New York), 301

Zavlin, V. E., Pavlov, G. G., \& Shibanov, Yu. A. (ZPS96) 1996 A\&A (in press)

Zimmermann, H.-U., et al. 1994, EXSAS Users's Guide, Edition 4, MPE Report 257, ROSAT Scientific Data Center, Garching

This paper is accepted for publication in The Astrophysical Journal Letters, 1996 